\title{
The Evaporation of Nearby Clouds and the Soft X-ray Background
}

\author{
Jonathan D. Slavin
}

NASA/Ames Research Center, MS 245-3, Moffett Field, CA 94035-1000, USA

\begin{abstract}
In recent years the nature of the low density clouds within the Local Bubble has been increasingly well characterized. These clouds, including the one which surrounds the solar system, are embedded in the hot gas and therefore should be evaporating via thermal conduction. If several evaporation fronts exist within the Local Bubble, the emissivity and spectrum of the hot gas is significantly different from a single temperature, equilibrium ionization plasma. We explore models in which the the temperature, density and ionization in the hot gas are influenced by cloud evaporation and compare the results with the observed Soft X-ray Background.
\end{abstract}

\section{Introduction}

In the past decade, it has become increasingly clear that the simplest model for soft x-ray emission from the Local Bubble (LB) is not adequate. Observations at lower energies (Be Band, EUVE MW spectrometer) have indicated that the spectrum is harder in the region of $50-100 \mathrm{eV}$ than the collisional ionization equilibrium, $T=10^{6} \mathrm{~K}$, Raymond \& Smith model predicts. Higher spectral resolution observations by $D X S$ (see Sanders, this volume) have confirmed that the simple models are not adequate to describe the emission.

At the same time, there has been mounting evidence that the Local Bubble, while of very low average density, does contain several low column density clouds (Frisch 1995 and references therein) including, of course, the Local Cloud in which the solar system is located. Warm $\left(T \approx 10^{4} \mathrm{~K}\right)$ clouds in a hot, soft $\mathrm{x}$-ray emitting region such as the Local Bubble should evaporate via thermal conduction unless a special magnetic field geometry exists which prevents it. There are now observational indications that at least some nearby clouds do indeed have evaporative interfaces at their boundaries (Gry et al. 1995, Bertin et al. 1995).

Evaporating clouds radiate strongly in the EUV/soft $\mathrm{x}$-ray spectral region. If the observed clouds in the LB are evaporating they could substantially alter the soft $\mathrm{x}$-ray spectrum which is observed. This could in turn alter our interpretation of the soft $\mathrm{x}$-ray background and potentially provide a solution to the puzzles of the SXRB spectrum. 


\section{Model}

We explore some simple models of a hot bubble which contains evaporating clouds. Our models of evaporating clouds are detailed, containing most physical effects which should be operating. These include saturation of thermal conduction, radiative cooling, and non-equilibrium ionization (see Slavin 1989). Our model of the bubble as a whole on the other hand is very simple. We model the average emission observed in the bubble as the combination of "ambient" hot gas at some temperature, $T_{f}$, and emission from evaporating cloud boundaries. The average observed intensity is:

$$
I_{E}=\left\langle\epsilon_{\mathrm{cl}}\right\rangle \ell_{b} f_{\mathrm{cl}}+\epsilon_{\mathrm{hot}} \ell_{b}\left(1-f_{\mathrm{cl}}\right),
$$

where $\left\langle\epsilon_{\mathrm{cl}}\right\rangle$ is the average emissivity in the cloud boundary regions, $\epsilon_{\text {hot }}$ is the emissivity in the ambient hot gas, $f_{\mathrm{cl}}$ is the cloud boundary filling factor and $\ell_{b}$ is the total path length in the bubble. The average emissivity for cloud boundary emission is just

$$
\left\langle\epsilon_{\mathrm{cl}}\right\rangle=\frac{\int_{0}^{R_{f}} \epsilon 4 \pi r^{2} d r}{\frac{4}{3} \pi R_{f}^{3}},
$$

where $\epsilon$ here is the emissivity at a given radial distance from a cloud center and $R_{f}$ is the cutoff radius. $R_{f}$ serves as an approximation to the mean distance between clouds (but due to cloud clustering, does not constrain $f_{\mathrm{cl}}$ ). We do not include the complex effects due to the interaction of the evaporative flows from adjacent clouds.

The cloud evaporation models have several parameters. These include the radius, $R_{\mathrm{cl}}$, temperature, $T_{\mathrm{cl}}$, density, $n_{\mathrm{cl}}$, and magnetic field, $B_{\mathrm{cl}}$, of the warm cloud and the reduction of conductivity due to magnetic field effects $\eta$. For this preliminary study we use the same values for these parameters in all the models: $R_{\mathrm{cl}}=3 \mathrm{pc}, T_{\mathrm{cl}}=10^{4} \mathrm{~K}, n_{\mathrm{cl}}=0.2 \mathrm{~cm}^{-3}, B_{\mathrm{cl}}=5 \mu \mathrm{G}$, and $\eta=1$.

Our models have two free parameters: $R_{f}$, the cutoff radius/mean cloud separation, and $T_{f}$, the temperature at $R_{f}$. We also use $T_{f}$ as the temperature of the "ambient" hot gas. The model results are compared with observations of the soft x-ray background (SXRB) in the Wisconsin Be and B bands. Integrating equation (1) over the detector band response function for each of these bands, and choosing a given observed value for the count rate in the two bands, results in two equations with two unknowns, $f_{\mathrm{cl}}$ and $\ell_{b}$ which we then solve for.

For our current purposes we have used the values of 22.5 and 0.275 counts $\mathrm{s}^{-1}$ for the $\mathrm{B}$ and Be band count rates. The $\mathrm{B}$ band value is typical of those observed near the galactic plane (McCammon et al. 1983). The Be band value is below those actually observed but results in a $\mathrm{Be} / \mathrm{B}$ band ratio which is the average of those observed (Bloch et al. 1986).

We calculate 12 models in all, one for $R_{f}=10,20$, and $30 \mathrm{pc}$ for each of $\log \left(T_{f}\right)=5.9,6.0,6.1$ and 6.2. Only for the models with $R_{f}=10 \mathrm{pc}$ do we find values which fall in the ranges: $0<f_{\mathrm{cl}}<1$ and $0<\ell_{b}<200 \mathrm{pc}$. 


\section{Results}

The parameters for our "successful" models are listed in Table 1. Even for models for which reasonable values of $\ell_{b}$ and $f_{\mathrm{cl}}$ are found, other considerations may rule them out. One such is the $\mathrm{C}$ band count rate. The $\mathrm{C} / \mathrm{B}$ band count rate ratio is confined to the range $1.9-3.3$. This is enough to exclude the $\log T_{f}=5.9$ model which has a ratio of 1.5. Another consideration is the path length. The $\log T_{f}=6.2$ model path length of 120 pc may weigh against it. Although the dimensions of the local HI cavity are generally uncertain, there are at least some directions which indicate a path length smaller than this. In the low column density direction of the third quadrant, however, the cavity appears to extend for a path length of up to $200 \mathrm{pc}$.

Table 1. Cloudy Bubble Model Results

\begin{tabular}{lccccccc}
\hline & & & & \multicolumn{4}{c}{ x-ray band count rate } \\
$\log T_{f}$ & $R_{f}(\mathrm{pc})$ & $\ell_{b}(\mathrm{pc})$ & $f_{\mathrm{cl}}$ & $\varepsilon_{\mathrm{cl}}^{\mathrm{Be}}$ & $\varepsilon_{\mathrm{cl}}^{\mathrm{B}}$ & $\varepsilon_{\text {hot }}^{\mathrm{Be}}$ & $\varepsilon_{\text {hot }}^{\mathrm{B}}$ \\
\hline 5.9 & 10 & 32 & 0.36 & 0.111 & 0.816 & 0.0604 & 0.657 \\
6.0 & 10 & 51 & 0.32 & 0.0786 & 0.570 & 0.0342 & 0.378 \\
6.1 & 10 & 80 & 0.43 & 0.0506 & 0.370 & 0.0161 & 0.214 \\
6.2 & 10 & 120 & 0.48 & 0.0354 & 0.250 & 0.00732 & 0.132 \\
\hline
\end{tabular}

${ }^{1}$ Emissivity integrated over the band (Be or B) effective area $\left(\mathrm{cm}^{2} \mathrm{sr}\right)$ in units of counts $\mathrm{s}^{-1} \mathrm{pc}^{-1}$ for the cloud boundary or "ambient" hot gas. For the cloud boundaries the emissivity is the average over the volume out to the cutoff radius, $R_{f}$. The thermal pressure in the hot gas ranges from approximately 9000 to $10^{4} \mathrm{~cm}^{-3} \mathrm{~K}$ in the models.

An example model spectrum, calculated using the Raymond \& Smith code (Raymond \& Smith 1977, and updates) is presented in Figure 1. The spectral region shown is that for which there is the most evidence of discrepancy with the uniform temperature, $T=10^{6} \mathrm{~K}$, equilibrium ionization model. The spectrum clearly does differ substantially from the uniform bubble model with the same $B$ band count rate. Particularly promising is the lower intensity of the iron line complex near $70 \mathrm{eV}$ which appears, both from Be band pulse height analysis (Bloch 1988) and EUVE observations (Vallerga \& Slavin, this volume) to be less than the uniform bubble model predicts.

\section{Summary}

We have created models in which Local Bubble contains clouds evaporating into the surrounding hot gas that match the soft $\mathrm{x}$-ray data. While these models are greatly oversimplified, they are a first step towards incorporating 


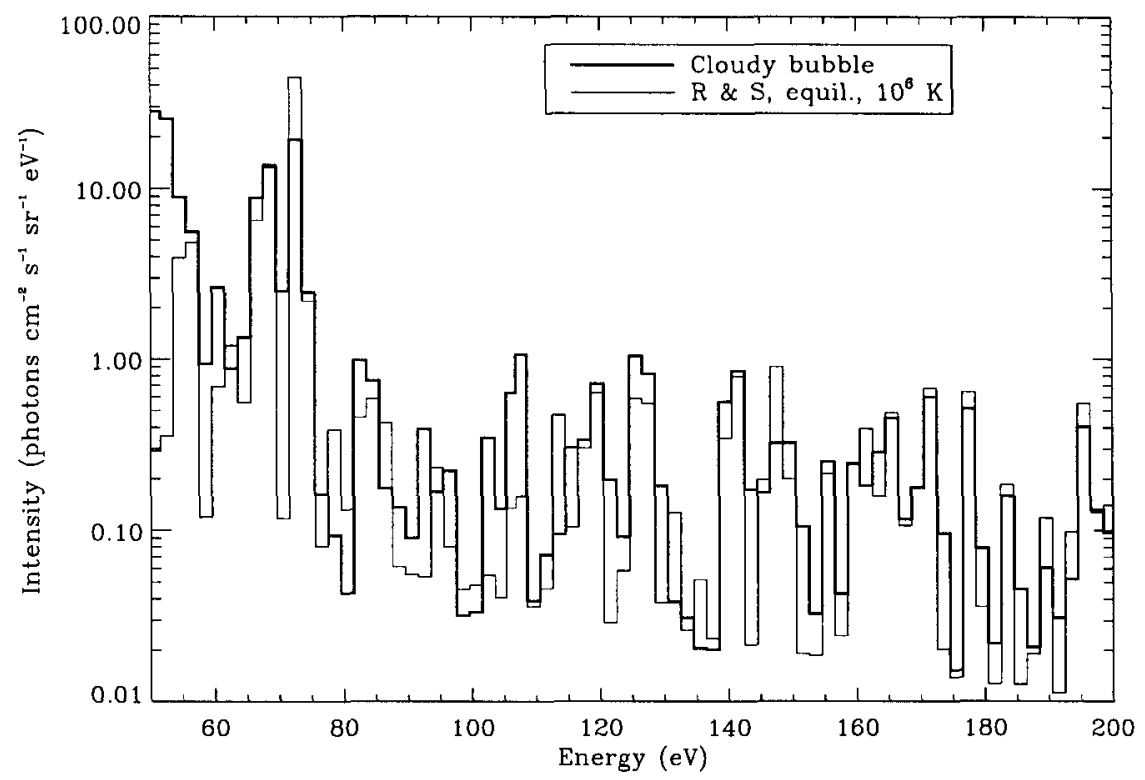

Fig. 1. An example of the low energy soft x-ray spectrum for a cloudy bubble model. For this model, $T_{f}=10^{6.1}$. The model contains emission from the boundary regions of evaporating clouds as well as ambient hot gas. The thick line is the spectrum of a Raymond \& Smith, equilibrium ionization, $T=10^{6} \mathrm{~K}$, model which produces the same $\mathrm{B}$ and $\mathrm{Be}$ band count rates as the cloudy bubble model.

the observed cloudy nature of the Local Bubble into models of the soft x-ray background emission.

\section{References}

Bertin, P., Vidal-Madjar, A., Lallement, R., Ferlet, R., \& Lemoine, M., 1995, A\&A 302,889

Bloch, J. J., Jahoda, K., Juda, M., McCammon, D., Sanders, W. T., \& Snowden, S. L. 1986, ApJ, 308, L59

Bloch, J. J. 1988, Ph.D. thesis, University of Wisconsin-Madison

Frisch, P. C. 1995, Space Sci. Rev., 72, 499

Gry, C., Lemonon, L., Vidal-Madjar, A., Lemoine, M., \& Ferlet, R. 1995, A\&A, 302,497

McCammon, D., Burrows, D. N., Sanders, W. T., \& Kraushaar, W. L. 1983, ApJ, 269, 107

Raymond, J. C. \& Smith, B. W. 1977, ApJS, 35419

Slavin, J. D., 1989, ApJ 346, 718 